\title{
Optimized DWT Based Digital Image Watermarking and Extraction Using RNN-LSTM
}

\author{
R. Radha Kumari ${ }^{1 *}$, V. Vijaya Kumar², K. Rama Naidu \\ ${ }^{1}$ Research Scholar, JNT University, Ananthpuramu (India) \\ ${ }^{2}$ Dean, Department of CSE \& IT and Director CACR, Anurag Group of Institutions, Hyderabad (India) \\ ${ }^{3}$ Professor, Department of ECE, Jawaharlal Nehru Technological University, Ananthpuramu (India)
}

Received 27 November 2020 | Accepted 21 August 2021 | Published 27 October 2021

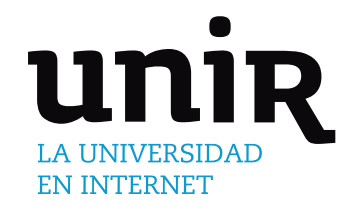

KEYWORDS

Discrete Wavelet Transform, Recurrent Neural NetworkLong Short-Term Memory, Simulated Annealing, Tunicate Swarm Algorithm, Watermarking.

OI: $10.9781 /$ ijimai.2021.10.006

\section{INTRODUCTION}

W ITH the rapid growth of digital data use and internet prevalent use, there are frequent acts of intellectual property infringement rights like copying, digital content theft and illegal use [1]. Digital images must be protected because they have high-value added contents for intellectual property rights. The digital watermarking technique is a recently developed technique to protect the digital images. In watermarking, the holder's information embeds the watermark into the content, which is then distributed or stored [2-3]. This topology claims the ownership by extracting embedded watermark information when needed. Numerous schemes have been researched based on the application side, technologies etc. Still, in recent times, various methods have been proposed to extract or modify the watermark algorithm along with the embedding process, to make it a watermark embedding algorithm [4]. In many areas, multimedia copyright protection has been protected by the watermarking techniques for security. Copyright protection is used for different applications like authentication, broadcast monitoring, cryptography and captioning.

To keep the audio, image and video info, the general information embedding technique of watermarking is to be used. It integrates the vital information into methods by invisibly altering the data [5]. Hence, robustness and invisibility are the two significant metrics for estimating the effectiveness of watermarking methods. The watermarking

${ }^{*}$ Corresponding author.

E-mail address: radharavada999@gmail.com techniques are also categorized into robust watermarking, fragile and semi fragile groups [6]. For data protection of image, the robust watermarking is important as it does not considerably decrease the watermarked image's visual quality and can tolerate numerous attacks. Hence, this is mostly utilized for owner verification and copyright protection. Fragile watermarking is used only to approve the entirety of the image without verifying the actual copyright [7]. For invisibility, the usual method embeds the watermark in DWT, Discrete Cosine Transform (DCT) and Discrete Fourier Transform (DFT) domain.

By comparing the spatial domain and the DCT based watermarking techniques, the DCT based watermarking approaches are robust. The algorithms of the DCT are robust in contrast to modest image processing tasks such as contrast adjustment, low-pass filtration, blurring and brightness, etc [8]. But DCT techniques are hard to implement and computationally expensive. They are also vulnerable to geometric attacks such as cropping, scaling, rotation, etc. It can be categorized into $8 \times 8$ block based DCT and global DCT watermarking [9]. Also it was affected by visual part of the image in the low-frequency sub band. Another fact is that the image's high-frequency components are normally detached by noise attacks and compression. Therefore the DCT watermark is embedded by changing the coefficients of the middle frequency sub bands [10]. DFT provides results in the amount of frequency content based on the phase and the magnitude. This is essential for the analysis of images and signals. DWT is, at present, in a variety of signal processing applications like noise mitigation in audio, simulation of wireless antenna distribution and audio and video compression [11]. On wavelets, basic functions are used to represent the signal. DWT is best suited for identifying the regions in the host 
image, where the watermark image can be embedded. Wavelets accumulate their energy over time, and they are very suitable for the analysis of transient and time varying signals.

The process of digital watermarking has three steps. In the first stage, the watermark is inserted in embedding. The second step is the attack step, where the degeneration of the watermarked image may or may not occur with a third-party attack. The last step is to find the original image, where the original image is retrieved from the watermarked image. Digital watermarking has been a moderately innovative field during the past two decades. All the digital info can be embedded in the data and then extracted [12]. The information of watermarking can be numbers, handwritten signatures, logos, texts, and can have many uses. It is important to note that one of the basic requirements for a digital watermark is to maintain a quality that does not distort the original data when a watermark is embedded in it. Besides, there are other necessities for specific applications [13].

In recent years, with the development of deep learning [14], convolution Neural Network (CNN) has made advances in the tasks of computer visions like semantic segmentation detection and classification [15]. In the field of super-resolution, the main feature of CNN-based methods is the ability to directly match the complex mapping amongst the low resolution and high resolution images, which enable a better retrieval of the lost high frequency information, and therefore its performance is beyond many classic approaches. Embedding performance is the probability of detection immediately after embedding [16]. While $100 \%$ performance is always desirable, it often comes at a very high cost in terms of other features. Depending on the application, one may be willing to sacrifice some performance for a better performance depending on some other characteristics [17].

The main contribution of the work is as follows.

- DWT is applied to protect the images from any attack in terms of embedding the watermark image into the original image.

- A hybrid optimization algorithm SA-TSA is presented to optimize the DWT coefficients on embedding strategy.

- The watermark image extraction process is performed based on a novel deep neural network concept called Recurrent Neural Network based Long Short-Term Memory (RNN-LSTM).

- The proposed method is evaluated and compared with existing methods like Artificial Neural Network (ANN), Deep Neural Network (DNN) and other optimization algorithms.

The rest of this paper is organized as follows: the recent research methods are given in section II. The proposed model description is mentioned in section III. The proposed work algorithm and performance analysis are given in sections IV and V respectively. To end with, the proposed work is concluded in the conclusion section.

\section{Related Works}

Some of the recent related works are discussed below.

Tanya and Azi [18] have suggested a blind image watermarking approach. The efficacy of the proposed scheme was enhanced by the hybrid Singular Value Decomposition (SVD) and DWT. DWT based 2-level SVD constraints were eliminated by this suggested approach. In watermarking, the watermark size depends on the cover image size. So, the suggested scheme is enhanced to be independent to form cover image sizes and watermark image sizes along with enhancing the imperceptibility and the robustness. For embedding the watermark, image blocking has to discover the optimal size of block and adjust the capability with the host image size. Before watermark embedding, additional pre-processing is done to achieve the goals. Based on the cover image size, the watermark image was discarded. The host and the watermark images were divided into $16 \times 16$ blocks. Furthermore, security was ensured by proposing a 2-level authentication method on behalf of extraction to discover the false negative and the false positive issues. The recommended method was tested and practiced for clinical \& non-clinical images revealing 10 kinds of geometric attacks.

In this digital period, illegal redistribution and the security of multimedia have become an important issue. Hence, digital watermarking has been presented to avoid the illegal activities and to ensure the authentication and security. Alotaibi [19] has formulated the video watermarking framework which includes video frame prediction by optimal, embedding and extraction processes. By utilizing the Jaya plus firefly algorithm, the optimal frames were selected based on the maximum Peak-signal to-noise-ratio (PSNR). Further frames are allotted with one or zero label, where zero indicates the decreased PSNR and one indicates the better PSNR. As a result, a data library was created since the results obtained, where every frame video was found by its gray-level co-occurrence matrix labels and features. The deep belief network was utilized to optimally select the frame by trained data which enhanced the prediction accuracy and end with watermark embedding and extraction.

In digital watermarking, the quality of digital content should not be compromised and it should not be visible to the human eye. Garg and Kishore [20] have offered a secure watermarking technique for color images. The Particle Swarm Optimization (PSO) algorithm was utilized in the watermarking process. Color watermark was utilized for embedding and embedded in R, G, and B planes of the color input image with entropy encryption and 2D-DCT. By using PSO, the embedding strength was optimized which offers a balance amongst the complexity and the strength. The suggested model was tested by various attacks on watermark image and the performances measures were NC and PSNR. With the intention of contributing to the medical image security, Kahlessenane et al. [21] have presented a robust watermarking approach which consents electronic patient record to be integrated into a computerized tomography scan. Before the integration process, the DWT was applied and the spatial rearrangement of LL subband coefficients was completed by the method of zig zag scanning. The gained coefficients were pooled to integrate the bits of the watermark. The integrity of watermark can be easily verified as it is integrated into the hash image of electronicpatient record. The experimental outcomes gave better PSNR results and good robustness for various attacks. Liu et al. [22] have proposed an image watermarking scheme by using DWT, SVD and Hessenberg decomposition (HD).

First, during the embedding progression, the input image was decomposed into multiple subbands by DWT, and the coefficients were used as HD input. At the same time, the watermark was functioned in the SVD. The watermark was lastly embedded by the scaling factor in the host image. The Fruit Fly optimization algorithm was dedicated for detecting the scaling factor via the objective function estimation process. Furthermore, the suggested technique was associated with the existing methods with various attacks like sharpening, JPEG compression, noise, filter and JPEG2000 compression and the results showed the robustness of the scheme. The transfer of patient record over the network requires a mechanism to ensure the privacy and security of the tele-health services. Ashima and Singh [23] have provided an advanced technique capable of protecting the data of the patients through embedding multiple watermarks on the medical image with the DWT-SVD domain. Before embedding, the hamming code was utilized in the text watermark to decrease the noise distortion on behalf of sensitive data. After the completion of embedding process, the watermarked image was extracted and compressed later. In the experiment, three compressions and two encryption schemes were tested. The outcomes demonstrated that the proposed method 
provided greater robustness compared to the other methods.

Digital watermarking applications are evidencing the digital content authenticity. For this concern, the watermarking methods were combined to the concepts of artificial networks and histogram. The histogram shape perception was put into practice, with the aim of maintaining the relevance and resistance of the watermark information within the host image. The optimization of the extraction process was proposed in [24]. The suggested artificial neural network was utilized to solve the strengthening resistance problems. The extraction progression was skilled by the auto encoder neural network and the back propagation neural network. The recommended method was checked under numerous attacks which gave better performances. The Firefly Algorithm (FA) is a newly created nature-inspired algorithm that is inspired by the luminous behavior of fireflies so that one firefly tends to attract other fireflies with greater brightness. The benefits of FA have automatic regrouping and local attractions. Hence, Guo et al. [25] has suggested FA based watermarking method with the help of DWT-QR transform. The objective function of the process has a bit error rate and Structural Similarity Index (SSIM). Experimental validation showed the invisibility property and the robustness.

The process of medical images denoising was considered to be a long established setback in the field of image processing. Rajeev et al. [26] have proposed an excellent system for denoising to eliminate salt \& pepper and white noises by relating LSTM or RNN and batch normalization technique. The input image of the suggested method was lung CT image. The PSO algorithm was utilized to calculate the batch size in effective way. RNN was utilized to denoise the image. LSTMbased Batch Normalization was presented to minimize the neural network's internal covariate-shift. The assessment matrices were MSE, Signal Noise Ratio or PSNR. The general idea of watermarking work was manipulating the color image underneath various attacks analyzed via the neural network tactic [27]. This was felt over the region of the transformation, particularly an emphasis on contourlet transform to discourse the suggested method, until the bands of the appropriate coefficients were precisely selected. On the color image, the logo information was embedded in the edges, whereas the Zenzo Edge detector was felt to handle the methodology. Actually, the margin of second sub-band was obtained, and then the capacity of the abovereferenced edge was computed.

The approaches to embedding and extracting during the learning process of the aforementioned neural network via the training data set are deliberated for continuous research with different scenarios in the current research. The proposed method's effectiveness is verified by the various scenarios analysis obviously.

\section{Proposed Watermarking Scheme}

The proposed digital image watermarking process is performed based on the process of embedding and extraction. In the process of embedding, the input image is applied to the DWT. In order to improve the robustness of the proposed technique, the DWT wavelet coefficients are optimized with the help of hybrid optimization technique. The SA algorithm is combined with TSA for better optimization. In embedding, the watermark image is converted into bits of watermarks for watermark embedding and to obtain the watermarked image. After performing the embedding process, the extraction is processed by deep neural network concept of Recurrent Neural Network based Long Short-Term memory. Finally, the watermark image and the original image are retrieved by this proposed RNN-LSTM approach. Fig. 1 illustrates the proposed scheme block diagram.

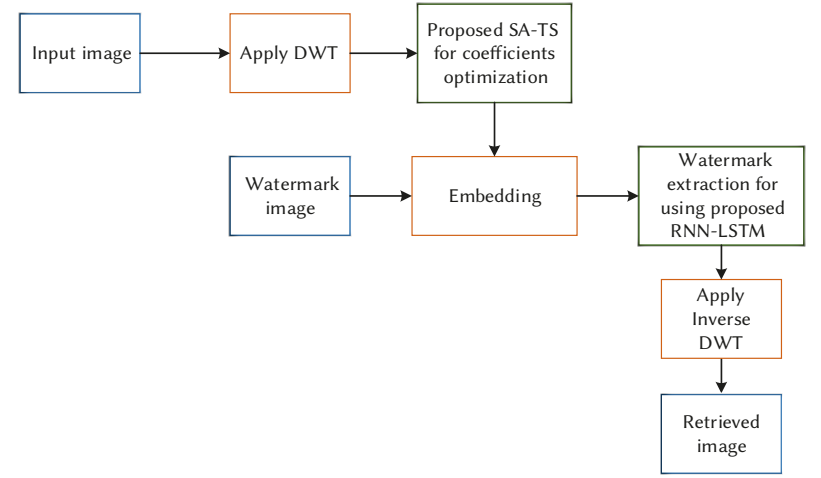

Fig. 1. Proposed Block Diagram.

\section{A. Watermark Embedding Process}

The proposed scheme has utilized the frequency domain; it is in the insertion of the tag, not directly into the image, however into its transformation. Therefore, before the integration, the DWT is applied into the image. The embedded watermarked image is obtained at the final stage of integration. The DWT process is explained below.

\section{Discrete Wavelet Transform (DWT)}

Many of the watermarking topologies are done by wavelets for its robustness and efficiency contrary to attacks. The image has a low frequency variation with fine details amongst high frequency variations. The low-frequency portion of DWT has high energy and it is more implored than the high frequency portion and separates the low-frequency components from the high-frequency components. In DWT, an image is decomposed into four types of subbands as LL approximation subband, $\mathrm{LH}, \mathrm{HH}$ and $\mathrm{HL}$ detail subbands.

Here, $\mathrm{L}$ indicates the low-pass filter and $\mathrm{H}$ indicates the highpass filter which is applied in individually as rows and columns. The choice of subband utilized on behalf of the watermark integration is influenced by the category of images used and its applications. If decomposition is completed, the input image is decomposed into four sub-bands. In contrast, the energy of HL, LH, and HH bands are low. For watermark embedding, the LL sub-band gives better results than the other. Different types of wavelet families are there which depends upon the mother wavelet choice. In our work, the DWT Haar filter is utilized by means of various decomposition levels so as to select the optimum DWT. The DWT is obtained by using the filter of Haar. The Haar filter is utilized to process the signals, and the coefficients of each sub-band is calculated by the equations (1), (2), (3) \& (4),

$$
\begin{aligned}
& L L(a, b)=\frac{p(a, b)+p(a, b+1)+p(a+1, b)+p(a+1, b+1)}{2} \\
& L H(a, b)=\frac{p(a, b)+p(x, y+1)+p(a+1, y)-p(a+1, b+1)}{2} \\
& H L(a, b)=\frac{p(a, b)-p(a, b+1)+p(a+1, b)-p(a+1, b+1)}{2} \\
& H H(a, b)=\frac{p(a, b)-p(a, b+1)-p(a+1, y)+p(a+1, b+1)}{2}
\end{aligned}
$$

Where, image sample pair is denoted as $a, b$. The transform of Haar wavelet decomposes the signal into two signals of half its length, one signal is running on average and other one is running on difference. Equation (5) describes the Haar wavelet mother function $\psi(t)$ :

$$
\psi(t)=\left\{\begin{array}{l}
1,0 \leq t<1 / 2 \\
-1,1 / 2 \leq t<1 \\
0, \text { otherwise }
\end{array}\right.
$$

The scaling function is described as in equation (6)

$$
\phi(t)=\left\{\begin{array}{l}
1,0 \leq t<1 \\
0, \text { otherwise }
\end{array}\right.
$$


Where, the scaling function and the Haar wavelet mother function are denoted as $\phi(t)$ and $\psi(t)$ respectively. The DWT is suitable for the watermark embedding process but the DWT operation enhancement is necessary. Hence, the DWT operation can be improved by manipulating the wavelet coefficients. In our work, the optimal wavelet coefficients are selected by using the proposed Hybrid optimization algorithm as SA-TSA. The following section explains the optimization process.

\section{Optimization Process}

In this article, we present the hybrid optimization approach for DWT wavelet coefficient optimization. The SA [28] and the TSA [29] are combined to form the new hybrid optimization algorithm called as SA-TSA. The TS algorithm is based on the behavior of tunicates for discovering the source of food and these activities are based on swarm intelligence and jet propulsion. Before starting the iteration process, the tunicates populations must be initialized. In search space, the TSA populations are randomly initialized. Here, we are using SA algorithm for initialization purpose, which is to enhance the speed of the convergence and the solution accuracy. SA must be applied for every individual of the initial population. In TS algorithm, the population of tunicates consists of $\vec{P}_{p}$. The objective function of this hybrid optimization is maximizing the PSNR of DWT.

The SA algorithm parameters such as the number of transitions and the temperature are represented as $N_{n}$ and $T_{n}$ respectively and $\mathrm{n}$ is denoted as iteration. The presented random number lies in between 0 and 1 . Hence, the optimization variable of the lower boundary and the upper boundary are indicated as $L_{b}$ and $H_{b}$ respectively. It is very important to highlight that each $x_{i}$ is a vector that is composed of variables that are being optimized. After the initialization, the tunicates swarm behavior and jet propulsion is applied to the whole population.

Basically, the swarm behavior and jet propulsion mechanism of tunicates occurs in four phases as,

i) Avoiding the conflicts among search agents

ii) Association towards the direction of best neighbor

iii) Converge to the finest search agent

iv) Swarm behavior

Step 1: After initialization, each search agent's fitness is calculated.

Step 2: The conflicts between the other tunicates can be avoided by employing the vector $\vec{K}$ as given in equation (7),

$$
\vec{K}=\frac{\vec{G}}{\vec{S}}
$$

Where, $\vec{G}=k_{2}+k_{3}-\vec{F}$ and $\vec{F}=2 . k_{1}$

Here, gravity force and water flow advection is represented as $\vec{G}$ and $\vec{F}$ respectively. The random variables of $k_{1}, k_{2}$, and $k_{3}$ lies in the range of zero to one and the social forces among the search agents are denoted as $\vec{S}$, which is given as in equation (8),

$$
\vec{S}=T_{\min }+k_{1} \cdot T_{\max }-T_{\min }
$$

Where, social interaction is to make the initial and the subordinate speeds which are denoted as $T_{\min }$ and $T_{\max }$ respectively. The values of $T_{\min }$ and $T_{\max }$ are one and four respectively.

Step 3: Distance between the food source and the search agent $(\vec{D})$ is calculated by equation (9),

$$
\vec{D}=\left|F_{S}-k \cdot \vec{P}_{p}(x)\right|
$$

Where, $x$ represents the present iteration and the food source location is $\vec{F}_{s}$ which is the optimal solution. The tunicate location is denoted as $\vec{P}_{p}^{s}(x)$
Step 4: Update the search agent position by equation (10),

$$
\overrightarrow{P_{P}}\left(x^{\prime}\right)=\left\{\begin{array}{l}
\overrightarrow{F_{S}}+\vec{K} \cdot \vec{D}, \text { if } k \geq 0.5 \\
\overrightarrow{F_{S}}-\vec{K} \cdot \vec{D}, \text { if } k<0.5
\end{array}\right.
$$

Where, updated tunicate position is denoted as $\vec{P}_{p}\left(x^{\prime}\right)$.

Step 5: Based on the tunicate swarm behavior, the optimal two solutions are stored and updated by the other search agent positions according to the greatest search agent position. The swarm behavior can be expressed as in equation (11),

$$
\vec{P}_{p}(x+1)=\frac{\vec{P}_{p}(x)+\vec{P}_{p}(x+1)}{2+k_{1}}
$$

Step 6: Updated search agent is adjusted beyond the boundaries in a given search location.

Step 7: The fitness of the updated search agent value is computed. If there is a better solution than the previous optimal solution, then update $P_{p}$.

Step 8: If the stopping criterion is reached, the algorithm will be stopped. Or else, repeat the steps 5-8.

Step 9: Return to the greatest optimal solution ever obtained.

Fig. 2 illustrates the proposed algorithm flowchart.

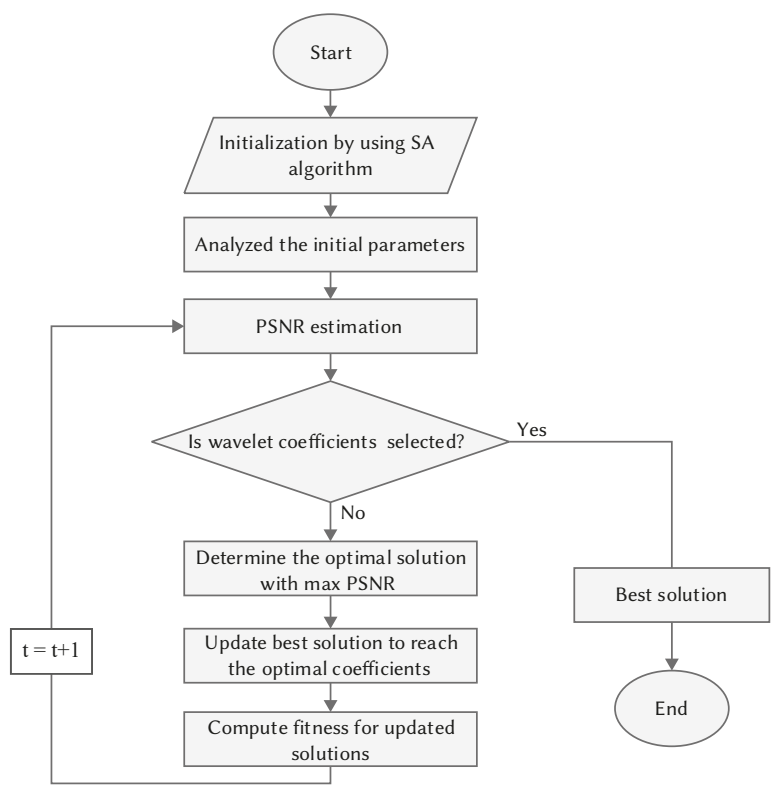

Fig. 2. Flow chart of the proposed SA-TS algorithm.

\section{Pseudo code for SA algorithm}

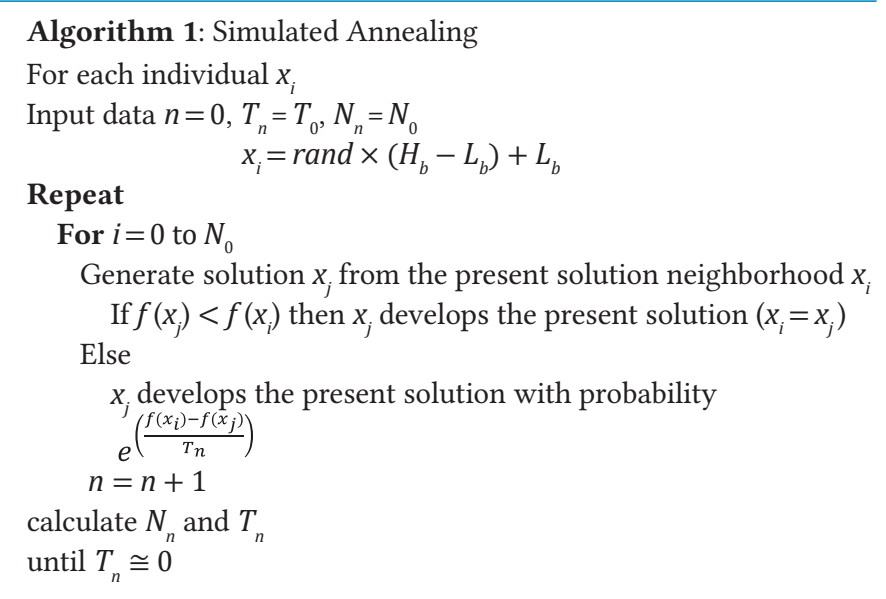


The following steps are to be used to embed the digital image watermark embedding:

Step 1: The original image is read at initially.

Step 2: DWT is applied into the input image.

Step 3: To obtain four types of sub bands, the one-level decomposition is performed.

Step 4: The optimized coefficients are obtained by the hybrid SATS algorithm.

Step 5: The watermark image is split into many blocks based on the pixel size $64 \times 64$.

Step 6: The watermark image is to be embedded and added to the information of sub-band.

Step 7: Obtained the watermarked image by the IDWT performance with an optimized DWT coefficients.

\section{A. Watermark Extraction Using Neural Network}

\section{Recurrent Neural Network}

An LSTM is designed to work differently than a CNN because an LSTM is usually used to process and make prediction on given sequence of data. During processing, the CNN completely loses all the information about the composition and position of the components and transmits the information further to a neuron which might not be able to extract the image. A Convolutional neural network is significantly slower due to an operation called maxpool. If the CNN has several layers then the training process takes a lot of time if the computer does not consist of a good GPU. Therefore, RNN-LSTM is better over the CNN technique [30].

The neural network of RNN has three layers as input, hidden and status layer. The input vector sequences are $x_{1}, x_{2}, \ldots, x_{T}$ and the hidden states sequences are $h_{1}, h_{2}, \ldots, h_{T}$ which are computed by step time $\mathrm{t}$ and expressed as in equation (12),

$h_{t}=\phi\left(\omega_{h} h_{t-1}+\omega_{x} x_{t}\right)$

Here, the recurrent weight matrix, the hidden weight matrix, and the arbitrary activation function are denoted as $\omega_{h}, \omega_{x}$ and $\phi$ respectively.

Finally, RNNs stack by inputting $\mathrm{h}$ into an additional different RNN, as a result creating deeper structures.

$$
h_{t}^{l}=\phi\left(\omega_{h} h_{t-1}^{l}+\omega_{x} h_{t}^{l-1}\right)
$$

In RNN, the sigmoid function of the activation function is represented by $\Phi$ which is a hyperbolic tangent. Networks' training is recognized as particularly difficult because the gradients are explodes and vanish. RNN has a specific memory function; however it cannot overcome the long-term dependency issues due to the complications of gradient explosion, gradient dispersion and RNN training. The LSTM network is a special RNN introduced by Schmidhuber and Hochreiter in 1997 [31]. The LSTM has solved the issues of longterm dependencies and continuously enhanced using the academic community. The LSTM's hidden layer arrangement is the long shortterm memory block, thereby the memory block contains cell structure and three thresholds control named as forget gate $\left(f_{t}\right)$, input gate $\left(i_{t}\right)$ and output gate $\left(\mathrm{o}_{\mathrm{t}}\right)$. By using the input vector $\left(\mathrm{h}_{\mathrm{t}-1}, \mathrm{x}_{\mathrm{t}}\right)$, the LSTM memory cell state is calculated by the forget gate.

$$
f_{t}=\sigma\left(\omega_{f} \cdot\left[h_{t-1}, x_{t}\right]+b_{f}\right)
$$

The new information generated in next part requires to be updated. This is considered as two stages; at first, the input gate computes the values throughout the used sigmoid function. Next, the new candidate values $c_{t}$ is generated by that layer, which is added into the memory cell.
Where, the current time input and the last time output are denoted as $x_{t}$ and $h_{t-1}$ respectively. The input layer bias and the weight are denoted as $\omega_{f}$ and $b_{f}$ and activation function is $\sigma($.$) which is generally$ known as sigmoid function.

$$
\begin{aligned}
& i_{t}=\sigma\left(\omega_{i} \cdot\left[h_{t-1}, x_{t}\right]+b_{i}\right) \\
& \tilde{c}_{t}=\tanh \left(\omega_{c} \cdot\left[h_{t-1}, x_{t}\right]+b_{c}\right. \\
& c_{t}=f_{t} * c_{t-1}+i_{t} * \tilde{c}_{t}
\end{aligned}
$$

Here, the status update layer bias and the weight function are denoted as $b_{c}$ and $\omega_{c}$ respectively.

Finally, the network is controlled by the updated state through the output gates in the output layer which is described in equation (18),

$$
\begin{aligned}
& o_{t}=\sigma\left(\omega_{o}\left[h_{t-1}, x_{t}\right]+b_{o}\right) \\
& h_{t}=o_{t} * \tanh \left(c_{t}\right)
\end{aligned}
$$

Where, the output layer bias and the weight are denoted as $b_{o}$ and $\omega_{o}$ respectively.

\section{B. Steps for Watermark Extraction}

Step 1: Forward DWT of the watermarked image taken.

Step 2: The image pixel blocks are selected.

Step 3: For the selected blocks, the middle value in each block is applied to RNN-LSTM.

Step 4: Based on the RNN-LSTM output and other some information, the watermark can be extracted from the watermarked image.

Step 5: Subtracted from the embedding reverse process by using the equation (20),

$$
\text { extrc_wtmk }=(e m b-m a t) /(4 * \mathrm{y}+2)+0.5(4.9)
$$

Where, y is denoted as neural network output and mat is the obtained matrix elements.

Step 6: Extract a watermark image and an original image.

Before extracting the watermark, it is important to distinguish among the watermarked and the original image. This should be done to check the watermarked image strength and compared to the original image. Subtraction of the original image from the watermarked image was finished in this connection. The watermark can be extracted by the second hidden layer of the neural network compared to the pixels of the distorted image pixel. Therefore the visible watermark is extracted by the proposed RNN-LSTM network.

\section{Results And Discussion}

The proposed work is implemented in the platform of MATLAB Intel core3 processor 2018b version. For the digital watermarking technique, the DWT was utilized to embed the watermark in the host image and the embedded watermark is robust against the various types of attacks. From the watermarked image, the original input image is extracted by the utilization of RNN-LSTM concept. For the experimental setup, the various test images are taken. The test images are peppers, Barbara, Lena and person and the image size is $512 \times 512$. We consider two watermark images; one is the copyright logo and another one is the cameraman image which has $64 \times 64$ pixel size. Fig. 3 and Fig. 4 show the original image and watermark image, respectively.

\section{Performance metrics}

To maintain the accuracy, we have used the following parameters as PSNR, Normalized coefficient (NC) and Mean Square Error (MSE). These matrices are evaluated in the proposed work. 


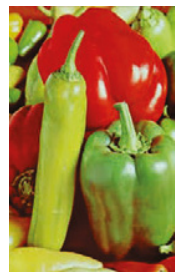

a) Peppers b) Barbara

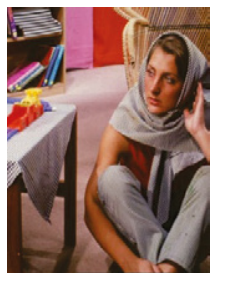

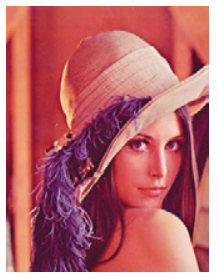

c) Lena

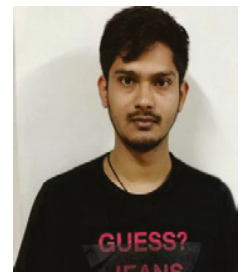

d) Person
Fig. 3. Original Image.

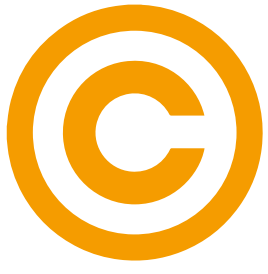

a) Copyright logo

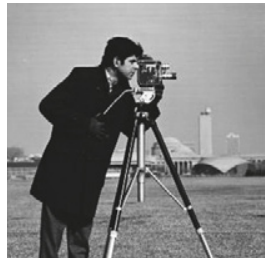

b) Cameraman
Fig. 4. Watermark Image

\section{Peak signal to noise ratio}

The watermarked image quality is measured by the parameter of PSNR. The ratio between the input image and the watermarked image is known as PSNR. Also the PSNR is measured based on the MSE. The PSNR is defined as in equation (21),

$$
P S N R=10 \log _{10}\left(\frac{255^{2}}{M S E}\right)
$$

\section{Normalized correlation}

It is used to evaluate the distance between the two vectors. It can be defined as in equation (22),

$$
N C=\sum_{a=1}^{n} \sum_{b=1}^{n} I_{(a, b)}^{i n} X O R I_{(a, b)}^{w} / n * n
$$

The input image is denoted as $I_{(a, b)}^{i n}$ and the watermarked image is denoted as $I_{(a, b)}^{w}$. For watermark bit generation, the Exclusive-OR (XOR) operation is used in watermark image embedding.

\section{Structural Similarity Index Measure}

The frame or image quality prediction method is called as SSIM. The SSIM is computed by the frame or images' various windows size. The SSIM is described as in equation (23),

$$
\operatorname{SSIM}(X, Y)=\frac{\left(\mu_{x} \mu_{y}+c_{1}\right)\left(2 \sigma_{x y}+c_{2}\right)}{\left(\mu_{x}^{2}+\mu_{y}^{2}+c_{1}\right)\left(\sigma_{x}^{2}+\sigma_{y}^{2}+c_{2}\right)}
$$

The input images' pixel size is $512 \times 512$ and the watermark images' pixel size is $64 \times 64$. The watermark image is converted into binary bits. By using the DWT, the decomposition is performed by the Haar filter. The DWT four level coefficients are optimized by the proposed SA-TSA algorithm. For each subsequent stage of wavelet decomposition, the previous level LL subband is utilized as input. At last, three levels of four sub-bands are attained, each band's pixel is $64 \times 64$. The host input image is reconstructed by the optimized DWT coefficient which is called as IDWT. Then the $64 \times 64$ pixel size is allocated into small blocks by $4 \times 4$ pixel sizes. For this, the wavelet transform incorporates the necessary features to attain the maximum advantages. Then the binary bits watermark image with $64 \times 64$ pixel is divided into non-overlapping small blocks with $4 \times 2$ pixel sizes, hence, $16 \times 32$ blocks are produced. After that, these blocks are inserted into the chosen wavelet coefficient blocks. Then the watermark image is embedded into block by block which reduces the processing time. The proposed image watermarking performance is tabulated in Table I.
For the extraction process, the trained RNN-LSTM network is to be used which is suitable for learning the relation amongst the watermarked image wavelet coefficients and the watermark image corresponding pixel. The decomposed watermarked image and its coefficients are split into $4 \times 4$ pixel small block dimensions. Then these coefficients' contents are extracted and utilized as the input of the trained RNN-LSTM and to achieve the watermarked data.

\section{A. Robustness of the Proposed Method}

Robustness is defined as the ability to attack variation without embracing the initial static formation of a system. In digital image watermarking scheme, robustness means the ability to extract the watermark from a watermarked image under several attacks. Hence, it is significant to identify the robustness of a watermarking system. To detect the proposed method performance, the two types of watermark images (watermark logo and cameraman) have been utilized to compare the robustness of the proposed techniques.

The recommended image watermarking approach has been evaluated for the outcomes and the robustness which are validated by the Tables II, III, IV and V respectively. The robustness of the RNNLSTM network based Peppers, Barbara, Lena and Person images' various attacked environments are presented. For the comparison, the performance matrices PSNR, NC and SSIM are measured.

SSIM is used to compare the watermarked images qualities after applying the attacks. For binary watermark, the NC is used to compare the robustness level. Table II describes the proposed RNN-LSTM network based method that achieves the highest NC value which is something that can be achieved. Therefore, the proposed method performs outstandingly by means of extracting the whole embedding watermark bits.

Table III describes the various attacks for Barbara image. At no attacks, the watermark image 1 PSNR is 56.005 and the watermark image 2 PSNR is 57.26. Thereby the cameraman watermark image performance has achieved a better PSNR. Table IV describes the various attacks for Lena image. At no attacks, the watermark image 1 PSNR is 56.004 and the watermark image 2 PSNR is 54.56. Thereby the copyright logo watermark image performance has achieved a better PSNR. In the comparison of robustness, the proposed method's performances are achieved with the no attacks, speckle noise, salt \& pepper noise, Gaussian noise, sharpening attack, rotation attack, motion blur, average filter, Jpeg compression, histogram equalization, rescaling and wiener filter.

Extracted watermark was affected by applying various attacks. If average filtering is applied, the $\mathrm{NC}$ value is close to 1 as 0.996 . Also, the image quality is affected, wherever the SSIM average values are nearer to 0.994 at watermark 1. In watermark 2, the NC is 0.9960 and SSIM is 0.9923. In the image watermarking, the high PSNR is achieved at no attacks as 56.004. When adding the speckle noise, Gaussian noise and salt \& pepper noise, the value of PSNR is decreased at 47.916, 46.30 and 38.503 respectively. In watermark image 2 , the PSNR value is 54.56 . Similarly, Table V describes the various attacks for Person image.

\section{B. Performance Evaluation}

In the proposed image watermarking scheme, from the watermarked image, the watermark image and the original image are extracted by using the RNN-LSTM network. The neural network approaches are suitable for this watermark extraction performance. After the attacks of cropping, rotation and JPEG the extracted images are partially degraded. Nevertheless, the extracted watermark images remain recognizable because these attacks change the indexed reference values, which may contain the watermark location values of the embedded values. If increases the RNN inputs, the watermark image extraction quality is degraded. This effect is seen because the 
TABLE I. Performance of the Proposed Image Watermarking

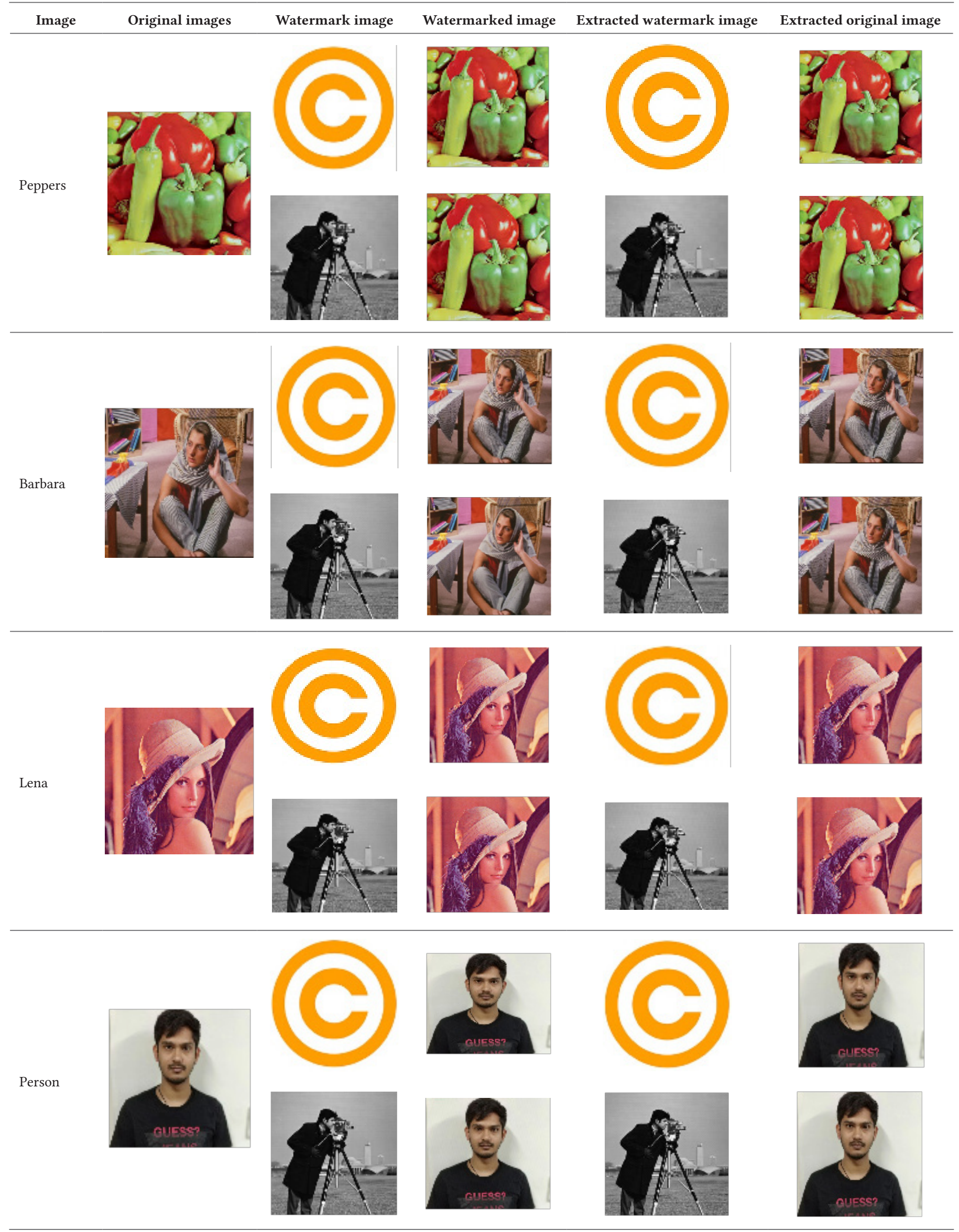


TABLE II. VARIous Attacks for Peppers Image

\begin{tabular}{|c|c|c|c|c|c|c|c|}
\hline \multirow{2}{*}{$\begin{array}{l}\text { Pepper } \\
\text { Image processing attacks }\end{array}$} & \multirow[b]{2}{*}{ Value } & \multicolumn{3}{|c|}{ Watermark image 1} & \multicolumn{3}{|c|}{ Watermark image 2} \\
\hline & & PSNR & NC & SSIM & PSNR & $\mathrm{NC}$ & SSIM \\
\hline No attack & - & 55.9949 & 0.9999 & 0.99718 & 61.0912 & 0.9995 & 0.9889 \\
\hline Speckle noise & 0.11 & 47.5906 & 0.9984 & 0.97876 & 41.2169 & 0.9999 & 0.9675 \\
\hline Salt \& pepper noise & 0.1 & 45.7953 & 0.9978 & 098667 & 39.9951 & 0.9961 & 0.9784 \\
\hline Gaussian noise & 0.002 & 38.6992 & 0.9987 & 0.86835 & 33.1583 & 0.9963 & 0.8466 \\
\hline Sharpening attack & 0.002 & 55.3312 & 0.9968 & 0.99606 & 45.234 & 0.9998 & 0.9878 \\
\hline Rotation attack & 0.002 & 50.8399 & 0.9912 & 0.99244 & 43.6997 & 0.9914 & 0.9844 \\
\hline Motion blur & 0.5 & 54.6194 & 0.9991 & 0.99632 & 47.828 & 0.9963 & 0.9950 \\
\hline Average filter & 0.8 & 54.195 & 0.9989 & 0.99281 & 47.5532 & 0.9978 & 0.9943 \\
\hline Jpeg compression & 50 & 53.6788 & 0.9996 & 0.99508 & 47.2182 & 0.9921 & 0.9936 \\
\hline Histogram equalization & 0.5 & 53.2366 & 0.9925 & 0.99247 & 43.114 & 0.9939 & 0.9800 \\
\hline Rescaling & 0.25 & 55.9949 & 0.9969 & 0.99718 & 45.3084 & 0.9984 & 0.9888 \\
\hline Wiener filter & 0.5 & 45.9911 & 0.9992 & 0.99788 & 45.3121 & 0.9971 & 0.9847 \\
\hline
\end{tabular}

TABLE III. VARIOUS AtTACKS FOR BARBARA IMAgE

\begin{tabular}{|c|c|c|c|c|c|c|c|}
\hline \multirow{2}{*}{$\begin{array}{l}\text { Barbara } \\
\text { Image processing attacks }\end{array}$} & \multirow[b]{2}{*}{ Value } & \multicolumn{3}{|c|}{ Watermark image 1} & \multicolumn{3}{|c|}{ Watermark image 2} \\
\hline & & PSNR & NC & SSIM & PSNR & NC & SSIM \\
\hline No attack & - & 56.0051 & 0.9989 & 0.99719 & 57.26 & 0.999 & 0.9822 \\
\hline Speckle noise & 0.11 & 48.0643 & 0.9985 & 0.97863 & 41.3977 & 0.9942 & 0.9688 \\
\hline Salt \& pepper noise & 0.1 & 46.3968 & 0.9950 & 0.98679 & 40.1401 & 0.9981 & 0.9781 \\
\hline Gaussian noise & 0.002 & 38.5094 & 0.9963 & 0.86404 & 32.9651 & 0.9957 & 0.8412 \\
\hline Sharpening attack & 0.002 & 55.4092 & 0.9959 & 0.95562 & 45.2324 & 0.9966 & 0.9880 \\
\hline Rotation attack & 0.002 & 42.6056 & 0.9928 & 0.95562 & 36.957 & 0.9909 & 0.9503 \\
\hline Motion blur & 0.5 & 54.1865 & 0.9900 & 0.99567 & 47.5454 & 0.9925 & 0.9942 \\
\hline Average filter & 0.8 & 52.974 & 0.9904 & 0.99381 & 46.6387 & 0.9932 & 0.9922 \\
\hline Jpeg compression & 50 & 46.0539 & 0.9915 & 0.973 & 40.4888 & 0.9947 & 0.9751 \\
\hline Histogram equalization & 0.5 & 52.565 & 0.9931 & 0.99289 & 42.6127 & 0.9937 & 0.9779 \\
\hline Rescaling & 0.25 & 54.2541 & 0.9907 & 0.99487 & 45.2619 & 0.9901 & 0.9887 \\
\hline Wiener filter & 0.5 & 50.4452 & 0.9933 & 0.9709 & 46.1529 & 0.9973 & 0.9805 \\
\hline
\end{tabular}

TABLE IV. VARIOUS ATTACKS FOR LENA IMAGE

\begin{tabular}{|c|c|c|c|c|c|c|c|}
\hline \multirow{2}{*}{$\begin{array}{l}\text { Lena } \\
\text { Image processing attacks }\end{array}$} & \multirow[b]{2}{*}{ Value } & \multicolumn{3}{|c|}{ Watermark image 1} & \multicolumn{3}{|c|}{ Watermark image 2} \\
\hline & & PSNR & NC & SSIM & PSNR & NC & SSIM \\
\hline No attack & - & 56.0045 & 0.9999 & 0.99719 & 54.5671 & 0.9999 & 0.9887 \\
\hline Speckle noise & 0.11 & 47.9165 & 0.9967 & 0.97779 & 41.2569 & 0.9951 & 0.9686 \\
\hline Salt \& pepper noise & 0.1 & 46.3007 & 0.9967 & 0.98652 & 40.225 & 0.9906 & 0.9776 \\
\hline Gaussian noise & 0.002 & 38.5032 & 0.9954 & 0.86394 & 32.9626 & 0.9975 & 0.8412 \\
\hline Sharpening attack & 0.002 & 55.4581 & 0.9964 & 0.99625 & 45.2069 & 0.9917 & 0.9878 \\
\hline Rotation attack & 0.002 & 45.277 & 0.9922 & 0.96869 & 39.3983 & 0.9999 & 0.958 \\
\hline Motion blur & 0.5 & 54.3272 & 0.9964 & 0.99576 & 47.6103 & 0.9996 & 0.9945 \\
\hline Average filter & 0.8 & 53.0465 & 0.9960 & 0.994 & 46.7244 & 0.9969 & 0.9923 \\
\hline Jpeg compression & 50 & 50.7845 & 0.9952 & 0.98998 & 44.8585 & 0.9960 & 0.9874 \\
\hline Histogram equalization & 0.5 & 50.8462 & 0.9996 & 0.99012 & 41.9573 & 0.9910 & 0.9732 \\
\hline Rescaling & 0.25 & 46.5957 & 0.9989 & 0.9934 & 46.6875 & 0.9909 & 0.9923 \\
\hline Wiener filter & 0.5 & 50.4436 & 0.9994 & 0.9970 & 45.2626 & 0.9988 & 0.9887 \\
\hline
\end{tabular}

TABLE V. Various Attacks for Real-Time Person Image

\begin{tabular}{|c|c|c|c|c|c|c|c|}
\hline \multirow{2}{*}{$\begin{array}{l}\text { Person } \\
\text { Image processing attacks }\end{array}$} & \multirow[b]{2}{*}{ Value } & \multicolumn{3}{|c|}{ Watermark image 1} & \multicolumn{3}{|c|}{ Watermark image 2} \\
\hline & & PSNR & $\mathrm{NC}$ & SSIM & PSNR & NC & SSIM \\
\hline No attack & - & 56.0052 & 0.9999 & 0.99718 & 55.6894 & 0.9999 & 0.9888 \\
\hline Speckle noise & 0.11 & 45.0682 & 0.9984 & 0.96322 & 39.0039 & 0.999 & 0.942 \\
\hline Salt \& pepper noise & 0.1 & 45.7215 & 0.9985 & 0.98741 & 39.8337 & 0.9962 & 0.9793 \\
\hline Gaussian noise & 0.002 & 38.5538 & 0.9925 & 0.86473 & 33.0254 & 0.9933 & 0.8428 \\
\hline Sharpening attack & 0.002 & 55.3819 & 0.9969 & 0.99615 & 45.2583 & 0.9905 & 0.988 \\
\hline Rotation attack & 0.002 & 49.7753 & 0.9981 & 0.99133 & 42.912 & 0.9961 & 0.9851 \\
\hline Motion blur & 0.5 & 54.8852 & 0.9952 & 0.99656 & 48.0028 & 0.9925 & 0.9955 \\
\hline Average filter & 0.8 & 54.6617 & 0.9947 & 0.99619 & 47.8321 & 0.9967 & 0.9952 \\
\hline Jpeg compression & 50 & 54.1889 & 0.9911 & 0.99542 & 47.4996 & 0.9931 & 0.9949 \\
\hline Histogram equalization & 0.5 & 50.0393 & 0.9936 & 0.99059 & 37.7344 & 0.9910 & 0.9335 \\
\hline Rescaling & 0.25 & 46.7290 & 0.9963 & 0.9872 & 45.9545 & 0.9978 & 0.9888 \\
\hline Wiener filter & 0.5 & 47.8321 & 0.9987 & 0.9953 & 45.2679 & 0.9981 & 0.9887 \\
\hline
\end{tabular}


higher the numbers of RNN-LSTM inputs indicate the fewer neural networks available for watermark extraction, i.e. lower the accuracy and computation time of RNN-LSTM. In watermarking, embedding and extraction computational complexity is necessary. The embedding strategy is easily and quickly performed but extraction is timeconsuming process. Excellent watermarks with high NC and PSNR values should be extracted for various aspects of watermarking algorithms, which prioritize image quality over speed. To analyses the effectiveness, the proposed RNN-LSTM neural network is compared with ANN [24] and DNN [32]. ANN, DNN and proposed RNN-LSTM are the deep neural network family techniques. Hence, the proposed method is compared with ANN and DNN. To show the proposed method efficiency, the PSNR, NC and SSIM values are calculated and compared with other deep neural networks.

From the comparison of Fig. 5, 6 and 7, it is clear that the value of proposed method PSNR is high compared to the other methods. Similarly, the value of NC and SSIM is higher than the existing methods. Therefore, RNN-LSTM assists better than the conventional methods in terms of PSNR, NC and SSIM. Table VI shows the measures of PSNR, NC and SSIM. The pepper image gives 55.994 db and 61.09 PSNR for watermark image 1 and watermark image 2. Similarly, for Barbara, Lena and person images, the PSNR value is higher than the other approaches. The NC metrics of the Pepper image value is 1 for watermark image 1 and watermark image 2, the RNN-LSTM NC value is 0.0998 which is higher than the existing methods. From the table VI, Barbara image SSIM value of proposed method is 0.9709 and existing method (ANN) is 0.965 and DNN is 0.965 . Likewise, pepper, Lena, and Person images' proposed SSIM value is higher than the existing methods. This performance is verified by the extracted watermark results, which demonstrates that the algorithm can maintain the quality of the watermarked image after embedding. The functional values of the image quality measurement confirm this result. Therefore, the watermarked image created by the proposed method has better imperceptibility than obtained using similar techniques because the PSNR values indicate that the watermarked image and the original image are identical.

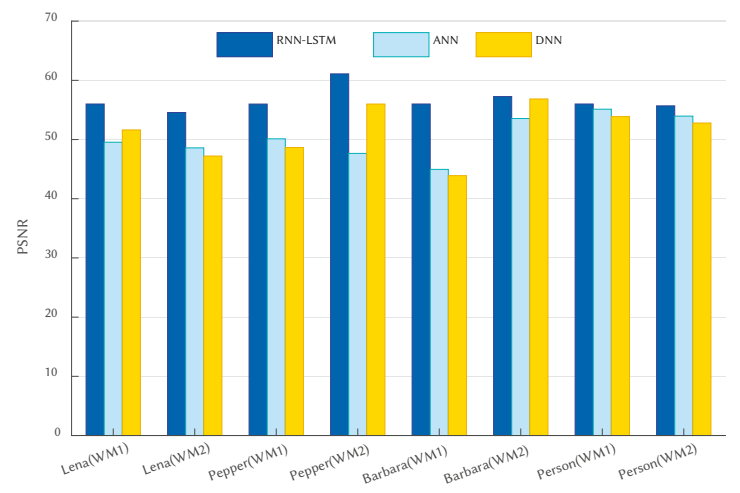

Fig. 5. Comparison of PSNR.

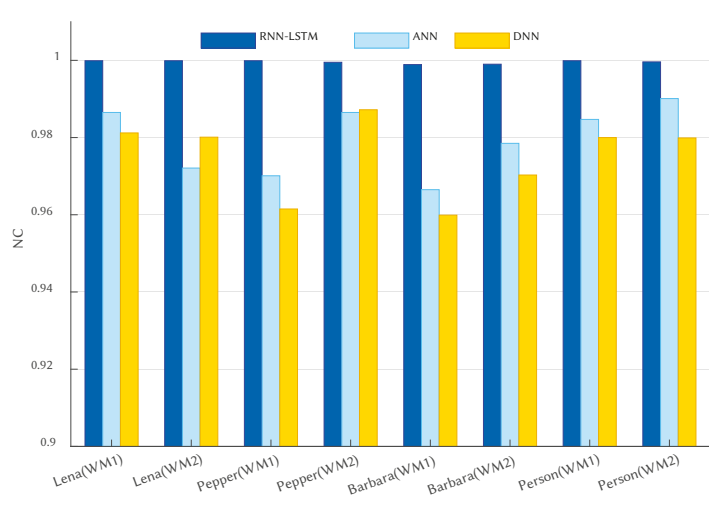

Fig. 6. Comparison of NC.

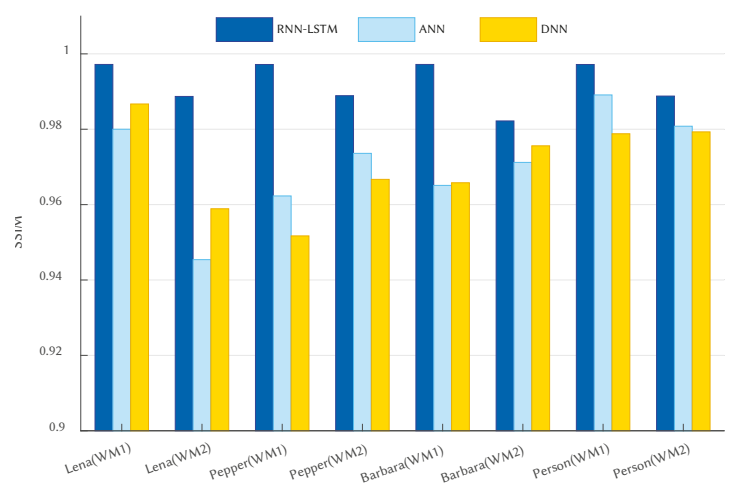

Fig. 7. Comparison of SSIM.

In the proposed work, the DWT wavelet coefficients are optimally selected by the proposed hybrid SA-TS algorithm. For a better optimal solution, we have utilized the hybrid optimization. Based on the maximum PSNR, the optimal coefficients are selected. The robustness of the algorithm is evaluated by the convergence plot of the proposed algorithm. Here the convergence graph is plotted with PSNR and its corresponding iterations. For the evaluation, the proposed SA-TSA is compared with the existing approaches of TSA and PSO [26]. The algorithm parameters are tabulated in Table VII. The three types of input images are taken and also two watermark images are utilized. So, the individual image PSNR convergence performances are displayed in the figures. Fig. 8 shows the Peppers image convergence graph for watermark image 1 and watermark image 2. By comparing the SATSA with PSO and TSA, the proposed method is achieving high PSNR (55.99) at watermark image 1 . In watermark image 2, the proposed PSNR is 61.09 which are higher than the existing algorithm as TSA (48.01) and PSO (47.23). If the number of iterations increases, the PSNR value is to be maintained constant.

TABLE VI. Comparison Graph of PSNR, NC and SSIM With Existing Techniques

\begin{tabular}{|c|c|c|c|c|c|c|c|c|c|c|}
\hline \multirow{2}{*}{ Image } & & \multicolumn{3}{|c|}{ RNN-LSTM } & \multicolumn{3}{|c|}{ ANN } & \multicolumn{3}{|c|}{ DNN } \\
\hline & & PSNR & NC & SSIM & PSNR & NC & SSIM & PSNR & NC & SSIM \\
\hline \multirow{2}{*}{ Peppers } & Watermark Img1 & 55.994 & 0.999 & 0.9971 & 50.122 & 0.970 & 0.962 & 48.659 & 0.961 & 0.951 \\
\hline & Watermark Img2 & 61.091 & 0.999 & 0.9889 & 47.658 & 0.986 & 0.973 & 55.998 & 0.987 & 0.966 \\
\hline \multirow{2}{*}{ Barbara } & Watermark Img1 & 56.005 & 0.998 & 0.9971 & 44.958 & 0.966 & 0.965 & 43.900 & 0.959 & 0.965 \\
\hline & Watermark Img2 & 57.26 & 0.999 & 0.982 & 53.552 & 0.978 & 0.981 & 56.854 & 0.970 & 0.975 \\
\hline \multirow{2}{*}{ Lena } & Watermark Img1 & 56.004 & 0.999 & 0.9919 & 49.548 & 0.986 & 0.98 & 51.624 & 0.981 & 0.986 \\
\hline & Watermark Img2 & 54.567 & 0.999 & 0.9887 & 48.593 & 0.972 & 0.945 & 47.214 & 0.980 & 0.958 \\
\hline \multirow{2}{*}{ Person } & Watermark Img1 & 56.005 & 0.999 & 0.9971 & 55.125 & 0.984 & 0.989 & 53.874 & 0.980 & 0.978 \\
\hline & Watermark Img2 & 55.689 & 0.999 & 0.9888 & 53.958 & 0.990 & 0.980 & 52.789 & 0.979 & 0.979 \\
\hline
\end{tabular}


TABLE VII. Algorithm Parameters

\begin{tabular}{lcc}
\hline Algorithms & Parameters & Values \\
\hline Proposed & Number of iterations & 100 \\
SA-TS & Number of search agents & Size of wavelet coefficient \\
& Number of population & 50 \\
Dimension & 5 \\
TSA & Fitness & Maximum PSNR \\
& Search agents & 20 \\
$I_{\text {min }}$ & 1 \\
PSO & Number of iterations & 4 \\
& Number of particles & 100 \\
& Inertia coefficient & 20 \\
& Cognitive \& social coefficient & 0.75 \\
& Number of iterations & $1.8 \& 2$ \\
& Number of hidden units & 100 \\
& Filter size & 120 \\
RNN-LSTM & Number of filters & 5 \\
& Dropout & 20 \\
& Mini batch & 0.1 \\
& Optimization & 320 \\
& Recurrent dropout & Adam \\
& Loss function & 0.1 \\
& & Cross entropy \\
& &
\end{tabular}

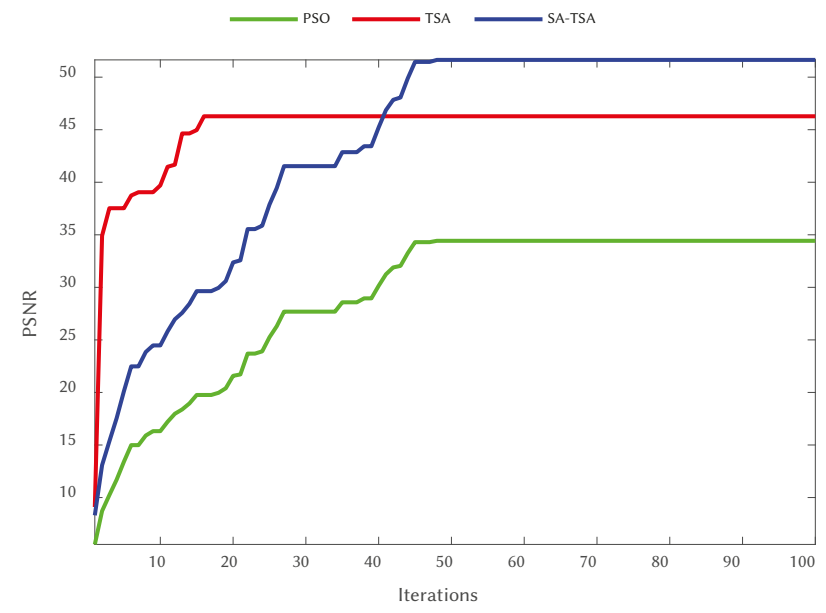

(a)
Fig. 9 shows the Barbara image convergence graph for watermark image 1 \& watermark image 2. By comparing the SA-TSA with PSO (40.07) and TSA (48.15), the proposed method is achieving high PSNR (56.005) at watermark image 1. In watermark image 2, the proposed PSNR is 57.26 which are higher than the existing algorithm as TSA (49.46) and PSO (42.56). Fig. 10 shows the Lena image convergence graph for watermark image 1 and watermark image 2. By comparing the SA-TSA with PSO (43.56) and TSA (45.08), the proposed method is achieving high PSNR (56.004) at watermark image 1. In watermark image 2, the proposed PSNR is 54.567 which are higher than the existing algorithm as TSA (50.10) and PSO (45.22). By the comparison, the suggested method gives a better PSNR than the other. Further, the watermark image 2 results are better than the watermark image 1 .

Fig. 11 shows the real-time Person image convergence graph for watermark image 1 and watermark image 2. By comparing the SA-TSA with PSO (35.46) and TSA (46.87), the proposed method is achieving high PSNR (56.005) at watermark image 1. In watermark image 2 , the proposed PSNR is 55.687 which are higher than the existing algorithm as TSA (47.93) and PSO (46.29). By comparison, the suggested method gives the better PSNR than the other. Further, the watermark image 2 results are better than the watermark image 1 .

From the outcomes, the recommended digital watermarking approach was performing very well in terms of robustness and

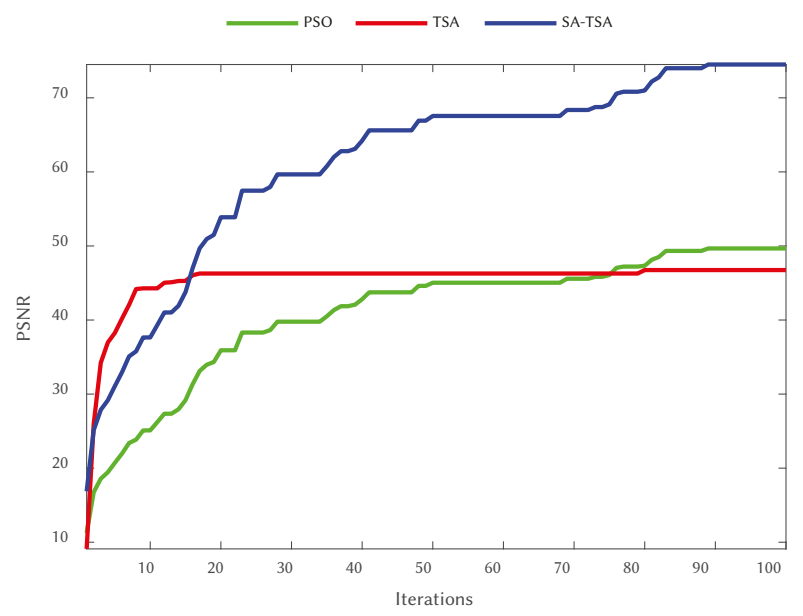

(b)

Fig. 8. Convergence for Peppers input image: a) watermarked image 1 and b) watermarked image 2.

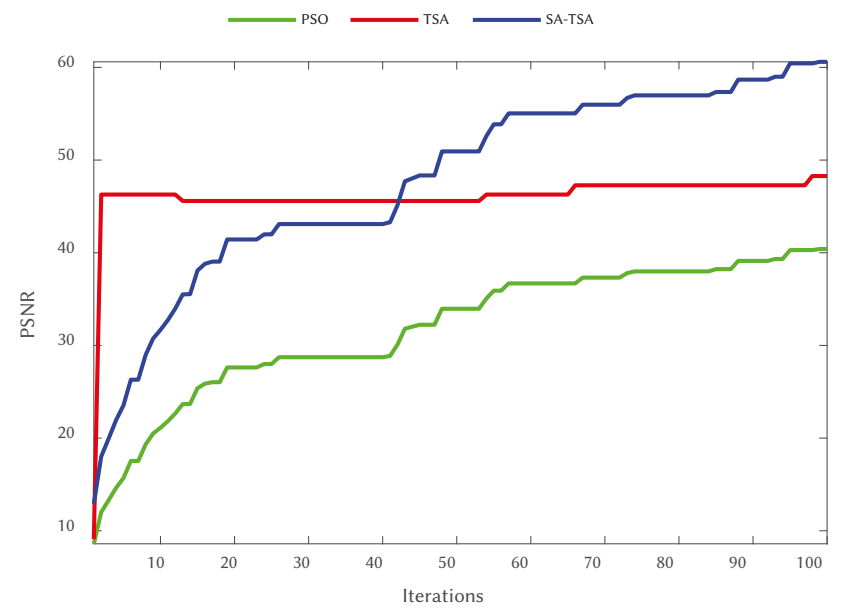

(a)

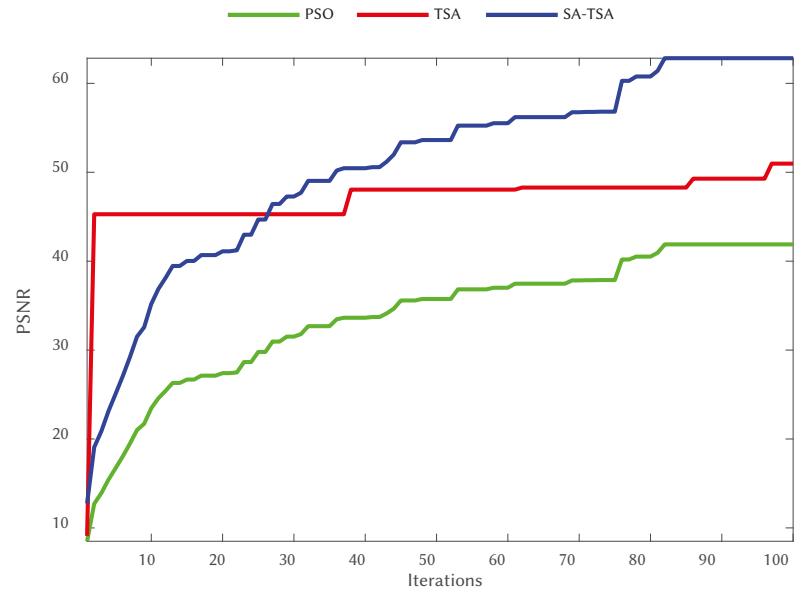

(b)

Fig. 9. Convergence for Barbara input image: a) watermarked image 1 and b) watermarked image 2. 
performance metrics. For the experimental assessment, the recommended method accomplishes more than 50dB PSNR which evaluates the quality of image watermarking. The robustness of the proposed model accomplished a good result by the SA-TS optimization algorithm and RNN-LSTM. Hence, the proposed method performances are verified in terms of perceptual quality which gives excellent results and achieves enhanced robustness performance.

\section{CONCLUSION}

The present research work focused on the development of an imperceptible and a robust non-blind image watermarking. The proposed digital image watermarking method uses DWT, SA-TS algorithm and RNN-LSTM for extraction to achieve the objectives. It is developed to embed a watermark in the host image without noticeable visual artifacts or degradation. The DWT coefficients are optimized by the proposed SA-TS algorithm. The watermark embedding is performed by DWT with SA-TS algorithm. The watermark extraction is done by the machine learning concept of RNN-LSTM. Four input images and two watermark images are provided in the experiment. Under various attacks, the results are achieved to show the robustness of the proposed work. In the comparison of robustness, the proposed method achieves better results with speckle noise, salt \& pepper noise, Gaussian noise, sharpening attack, rotation attack, motion blur, average filter, Jpeg compression, histogram equalization, rescaling and wiener filter. The proposed optimization algorithm performance is compared with TSA and PSO algorithm. From the convergence, the maximum PSNR is achieved by the proposed algorithm. Also, the RNNLSTM results are compared with the measures like PSNR (avg58.542), NC (0.999) and SSIM (avg0.992) with DNN and ANN. From an overall perspective, this technique can bring substantial benefits to the field of digital watermark and additional benefits for copyright protection. In the future work, deep learning network will be used in both embedding and extraction step. Additionally, the image preprocessing approach is used to enhance watermark image quality.

\section{REFERENCES}

[1] I. Hamamoto, M. Kawamura. "Image watermarking technique using embedder and extractor neural networks." IEICE TRANSACTIONS on Information and Systems, Vol.102, No. 1, January 2019, pp. 19-30. DOI:https://doi.org/10.1587/transinf.2018MUP0006.

[2] X. Zhou, H. Zhang, and C. Wang. "A robust image watermarking technique based on DWT, APDCBT, and SVD.” Symmetry, Vol .10, No. 3, March 2018, pp. 77. DOI:https://doi.org/10.3390/sym10030077.

[3] Z. Renjie, X. Zhang, M. Shi, and Z. Tang. "Secure neural network

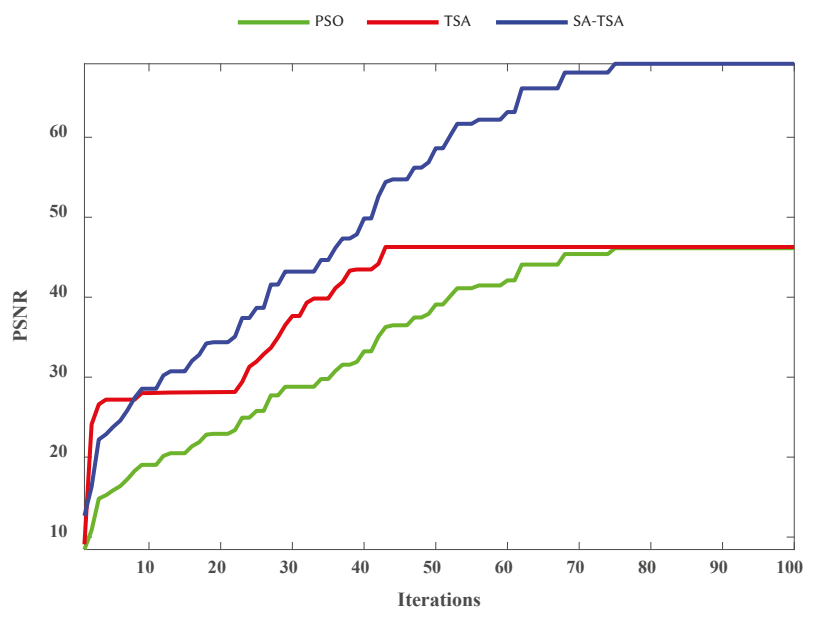

(b)

(a)

Fig. 10. Convergence for Lena input image: a) watermarked image 1 and b) watermarked image 2.

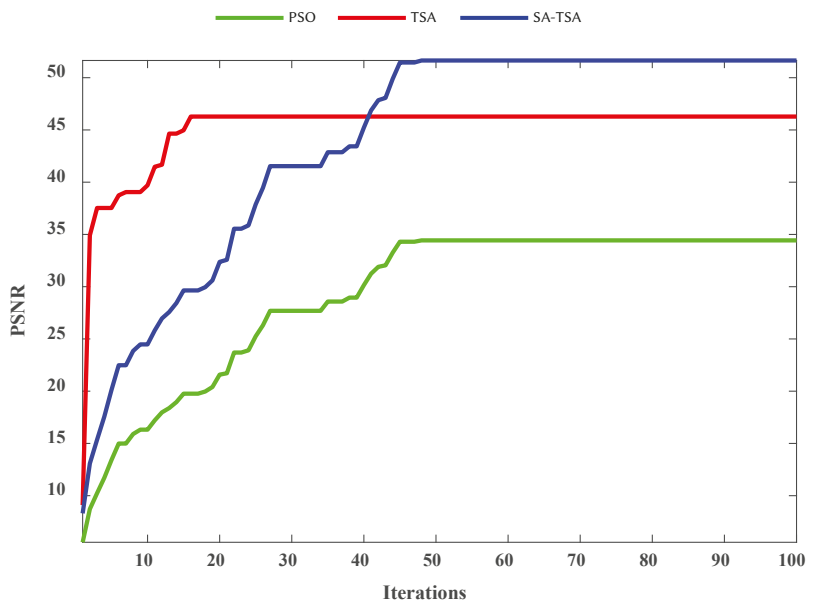

(a)

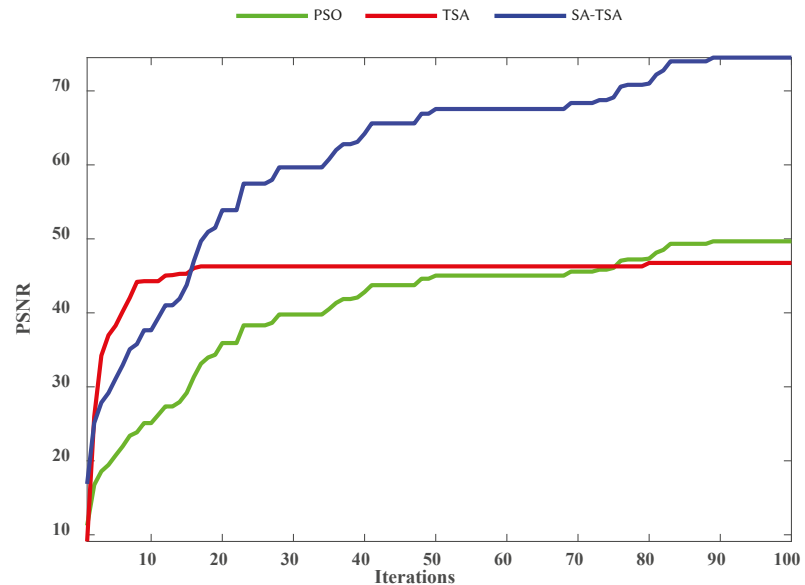

(b)

Fig. 11. Convergence for Person input image: a) watermarked image 1 and b) watermarked image 2 . 
watermarking protocol against forging attack." EURASIP fournal on Image and Video Processing, September 2020, pp. 1-12. DOI: https://doi. org/10.1186/s13640-020-00527-1.

[4] S.P. Ambadekar, J. Jain, and J. Khanapuri. "Digital image watermarking through encryption and DWT for copyright protection." In Recent Trends in Signal and Image Processing, Vol.727, May 2018, pp. 187-195.

[5] T.K Araghi, A. A Manaf. "An enhanced hybrid image watermarking scheme for security of medical and non-medical images based on DWT and 2-D SVD." Future Generation Computer Systems Vol.101, December 2019, pp. 1223-1246. DOI: 10.1016/j.future.2019.07.064.

[6] M. Ali, and C. W. Ahn. "An optimal image watermarking approach through cuckoo search algorithm in wavelet domain." International fournal of System Assurance Engineering and Management, Vol.9, No. 3, June 2018, pp. 602-611. DOI: 10.1007/s13198-014-0288-4.

[7] D. Rajani, and P. Rajesh Kumar. "An optimized blind watermarking scheme based on principal component analysis in redundant discrete wavelet domain." Signal Processing, Vol. 172, July 2020, pp.107556. DOI: https://doi.org/10.1016/j.sigpro.2020.107556.

[8] X. Kang, F. Zhao, G. Lin, and Y. Chen. "A novel hybrid of DCT and SVD in DWT domain for robust and invisible blind image watermarking with optimal embedding strength." Multimedia Tools and Applications, Vol 77, No. 11, July2017, pp. 13197-13224. DOI:https://doi.org/10.1007/s11042017-4941-1.

[9] P. Kadian, N. Arora, and S. M. Arora. "Performance Evaluation of Robust Watermarking Using DWT-SVD and RDWT-SVD." In 2019 6th International Conference on Signal Processing and Integrated Networks (SPIN) IEEE, May 2019, pp. 987-991.

[10] A.K. Abdulrahman, and S. Ozturk. "A novel hybrid DCT and DWT based robust watermarking algorithm for color images." Multimedia Tools and Applications, Vol. 78, no. 12, January 2019, pp. 17027-17049, DOI:https:// doi.org/10.1007/s11042-018-7085-z.

[11] F.N. Thakkar, and V. K. Srivastava. "Performance comparison of recent optimization algorithm Jaya with particle swarm optimization for digital image watermarking in complex wavelet domain." Multidimensional Systems and Signal Processing, Vol. 30, no. 4, 2019, pp. 1769-1791.

[12] M. Ahmadi, A. Norouzi, N. Karimi, S. Samavi, and A. Emami. "ReDMark: Framework for residual diffusion watermarking based on deep networks." Expert Systems with Applications, Vol.146, May 2020 pp.113157.

[13] F. López, L. de la Fuente Valentín, and I. S. M. de Mendivil. "Detecting image brush editing using the discarded coefficients and intentions." International fournal of Interactive Multimedia and Artificial Intelligence, Vol. 5, no. 5, 2019, pp.15-21.

[14] N. Saleem, and M. I. Khattak. "Deep Neural Networks for Speech Enhancement in Complex-Noisy Environments." International fournal of Interactive Multimedia and Artificial Intelligence, Vol. 6, no. 1, 2020, pp. 84-90.

[15] J.E. Lee, Y.H. Seo, and D.W. Kim. "Convolutional Neural Network-Based Digital Image Watermarking Adaptive to the Resolution of Image and Watermark." Applied Sciences, Vol.10, no. 19, September 2020, pp. 6854. https://doi.org/10.3390/app10196854.

[16] Y.Nagai, Y.Uchida, S.Sakazawa, and S. Satoh. "Digital watermarking for deep neural networks." International fournal of Multimedia Information Retrieval, Vol 7, no. 1, February 2018, pp. 3-16. DOI:https://doi. org/10.1007/s13735-018-0147-1.

[17] F. Wang, X. Liu, G. Deng, X. Yu, H. Li, and Q.Han. "Remaining life prediction method for rolling bearing based on the long short-term memory network." Neural Processing Letters, Vol. 50, no. 3, March 2019, pp2437-2454. DOI: 10.1007/s11063-019-10016-w.

[18] T.K. Araghi, and A. A. Manaf. "An enhanced hybrid image watermarking scheme for security of medical and non-medical images based on DWT and 2-D SVD." Future Generation Computer Systems Vol. 101, December 2019, pp. 1223-1246.DOI: 10.1016/j.future.2019.07.064

[19] S.S. Alotaibi, "Optimization insisted watermarking model: hybrid firefly and Jaya algorithm for video copyright protection." Soft Computing, Vol.24, March 2020, pp. 14809-14823. DOI:https://doi.org/10.1007/s00500020-04833-8.

[20] P. Garg, and R.R.Kishore. "Optimized color image watermarking through watermark strength optimization using particle swarm optimization technique." Journal of Information and Optimization Sciences Vol.41, No.6, 2020, pp1-14.
[21] F. Kahlessenane, A. Khaldi, R. Kafi, and S.Euschi. "A DWT based watermarking approach for medical image protection." fournal of Ambient Intelligence and Humanized Computing, August 2020, pp1-8.

[22] J. Liu, Huang, Y. Luo, L.Cao, S. Yang, D.Wei, and R. Zhou. "An optimized image watermarking method based on HD and SVD in DWT domain." IEEE Access, Vol. 7, May 2019, pp. 80849-80860. DOI: 10.1109/ ACCESS.2019.2915596.

[23] A. Ashima, and A.K. Singh. "An improved DWT-SVD domain watermarking for medical information security." Computer Communications Vol. 152, February 2020, pp.72-80. DOI: https://doi. org/10.1016/j.comcom.2020.01.038.

[24] R.R. Sunesh Kishore, and A. Saini. "Optimized image watermarking with artificial neural networks and histogram shape." fournal of Information and Optimization Sciences, Vol .44, No.7, September 2020, pp. 1597-1613.

[25] Y. Guo, B-Z. Li, and N.Goel."Optimised blind image watermarking method based on firefly algorithm in DWT-QR transform domain." IET Image processing, Vol.11, No. 6, June 2017, pp.406-415. DOI: 10.1049/ietipr.2016.0515.

[26] R. Rajeev, J. Abdul Samath, and N. K. Karthikeyan. "An Intelligent Recurrent Neural Network with Long Short-Term Memory (LSTM) BASED Batch Normalization for Medical Image Denoising." Journal of medical systems, Vol. 43, No. 8 , June 2019,pp 234. https://doi.org/10.1007/ s10916-019-1371-9.

[27] M.F. Kazemi, M. A. Pourmina, and A. H. Mazinan. "Analysis of watermarking framework for color image through a neural networkbased approach." Complex \& Intelligent Systems, Vol.6, January2020, pp.213-220. https://doi.org/10.1007/s40747-020-00129-4.

[28] N. Leite, F. Melício, and A.C. Rosa. "A fast simulated annealing algorithm for the examination timetabling problem." Expert Systems with Applications, Vol122, May2019, pp. 137-151. DOI:https://doi.org/10.1016/j. eswa.2018.12.048

[29] S. Kaur, L.K. Awasthi, A. L. Sangal, and G. Dhiman. "Tunicate Swarm Algorithm: A new bio-inspired based metaheuristic paradigm for global optimization." Engineering Applications of Artificial Intelligence Vol. 90, pp. 103541. https://doi.org/10.1016/j.engappai.2020.103541.

[30] W. Ding, Y. Ming, Z. Cao, and C.T. Lin. "A Generalized Deep Neural Network Approach for Digital Watermarking Analysis. "IEEE Transactions on Emerging Topics in Computational Intelligence. 2021.

[31] S. Hochreiter and J. Schmidhuber. "Long short-term memory". Neural computation, Vol.9, no. 8, 1997, pp. 1735-1780.

[32] H.W. Makram, J-F. Couchot, R. Couturier, and R. Darazi. "Using Deep learning for image watermarking attack." Signal Processing: Image Communication, Vol. 90, October2020, pp.116019. https://doi. org/10.1016/j.image.2020.116019.

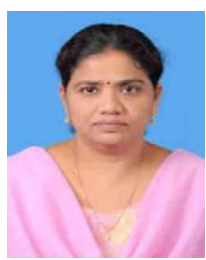

R. Radha Kumari

R. Radha Kumari working as Associate Professor in SACET (Affiliated to JNT University, Kakinada), Chirala, India, and she has 17 years of teaching experience. She has received M.Tech. Degree in VLSI Design from Sathyabama university, Chennai and B.Tech in Electronics \& Communication Engineering from N.B.K.R.I.S.T, Vidyanagar, Nellore(dt) A.P. Her areas of interest includes

VLSI Design, Digital image processing and Artificial Intelligence, Machine Learning.

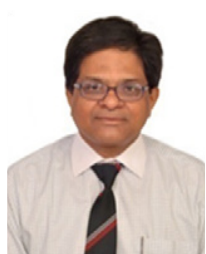

\section{Dr. V. Vijaya Kumar}

Dr. V. Vijaya Kumar is working as Professor \& Dean Department of Computer Science \& Engineering and Information technology in Anurag Group of Institutions (Autonomous) Hyderabad. $\mathrm{He}$ received integrated M.S.Engg, in CSE from USSR in 1989. He received his Ph.D. from Jawaharlal Nehru Technological University (JNTU), Hyderabad, India in 1998 in CSE and guided 33 research scholars for Ph.D. He acted as principle investigator for various R\&D projects. He has served JNT University for 13 years as Assistant Professor, Associate Professor and Professor. He has received Distinguished Professor award from Computer Science of India (CSI), Mumbai, best researcher and best teacher award from JNT University, Kakinada, India, Leading Scientist of the WORLD -2009 and Top 100 Scientists award in 2010 from International 
Biographical Centre, Cambridge, England. His research interests include Big data and image analytics, image retrieval, texture analysis, author attribution, digital water marking. At present he is also acting as BoS member for various universities and institutions. He is the life member of CSI, ISCA, ISTE, IE (I), IETE, ACCS, CRSI, IRS and REDCROSS. He published more than 120 research publications till now in various national, international journals and conferences. He has delivered key note addresses at various international conferences.

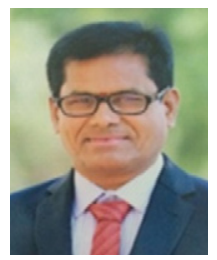

Dr. K. Rama Naidu

Dr. K. Rama Naidu obtained his B.Tech (ECE) from JNTU College of Engineering, Anantapur in 1983 and M.Tech (Microwave Engineering) from Institute of Technology, Banaras Hindu University, 1990. He obtained his Ph.D. (Wireless Communications) from Indian Institute of Technology, Kharagpur in 2009. His areas of research are channel modelling, resource allocation for wireless relay systems, PAPR reduction in OFDM, channel estimation, and cognitive radios. He has published and presented more than 28 technical papers in various International Journals \& conferences. He has 29 years of experience in teaching. Currently he is working as Professor, Dept. of ECE, at JNTUA College of Engineering, Ananthapuramu, A. P. 